\title{
ORTHOGONAL HILBERT TRANSFORM FILTER BANKS AND WAVELETS
}

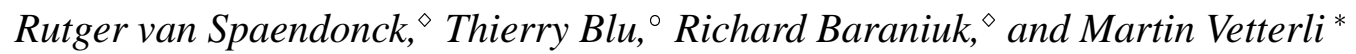 \\ ${ }^{\diamond}$ Department of Electrical and Computer Engineering, Rice University, Houston, USA \\ ${ }^{\circ}$ BIG and * LCAV, EPFL, Lausanne, Switzerland
}

\begin{abstract}
Complex wavelet transforms offer the opportunity to perform directional and coherent processing based on the local magnitude and phase of signals and images. Although denoising, segmentation, and image enhancement are significantly improved using complex wavelets, the redundancy of most current transforms hinders their application in compression and related problems. In this paper we introduce a new orthonormal complex wavelet transform with no redundancy for both real- and complex-valued signals. The transform's filterbank features a real lowpass filter and two complex highpass filters arranged in a critically sampled, threeband structure. Placing symmetry and orthogonality constraints on these filters, we find that each high-pass filter can be factored into a real highpass filter followed by an approximate Hilbert transform filter.
\end{abstract}

\section{INTRODUCTION}

What value do complex wavelets add over conventional realvalued wavelets? The answer is that they contain both amplitude and phase information, just the type of information needed to accurately describe the energy localization of oscillating functions. Wavelets are oscillating functions. As a consequence, edges and other singularities manifest themselves in the wavelet domain as oscillating coefficients. The amplitudes of these coefficients describe the strength of the singularity while the phase indicates the location of the singularity.

In order to determine the envelope and phase of an oscillating function, we generally use an analytic signal or quadrature representation, which can be obtained from the Hilbert transform of the signal. Due to its global character, the Hilbert transform is not suited to be mixed with local wavelets. This observation points to the need for local filters with properties similar to a Hilbert transform. For higher-dimensional signals such as images, the Hilbert transform property of complex wavelets can be used to improve the angular sensitivity of the transform.

There is no one unique extension of the conventional real wavelet transform into the complex plane. Lawton [1] and later Lina [2] were the first to exploit the complex solutions of Daubechies wavelet filters. These result in symmetrical orthogonal filter pairs, a combination of beneficial properties that cannot be obtained with real wavelets. However, the filters lack quadrature between their real and imaginary parts, a desirable property especially for the interpretation of phase. Kingsbury [3] designed the dual-tree complex wavelet transform to

$\diamond$ This work was partially supported by NSF, ONR, AFOSR, DARPA, Texas Instruments and the Rice Consortium for Computational Seismic Interpretation. E-mail: \{rutger,richb\}@rice.edu, $\{$ martin.vetterli,thierry.blu\}@epfl.ch, www.dsp.rice.edu

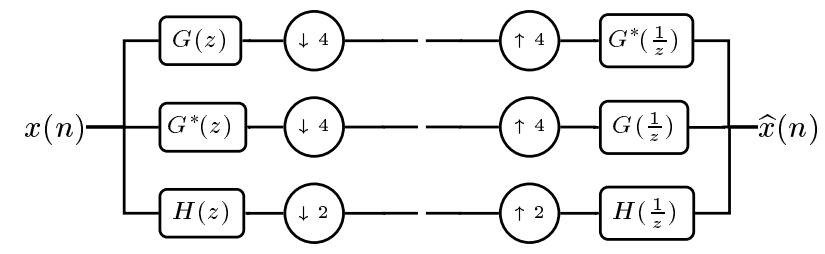

Fig. 1. Orthoconjugate filter bank structure.

improve the shift-invariance of the wavelet transform. This redundant transform consists of two wavelet filter bank trees using filters in approximate quadrature. Selesnick [4] refined the dual-tree transform such that in the limit the scaling and wavelet functions form Hilbert transform pairs. The redundancy of all of the above transforms is however a drawback for applications like compression. Therefore van Spaendonck et al. [5] created a set of projection-based, non-redundant transforms. While these transforms are restricted to IIR filters, Fernandes et al. [6] showed that they admit orthogonal solutions.

In this paper, we propose a new complex wavelet transform based on the one-dimensional (1-d), three-band, complex filterbank shown in Fig. 1. This structure arises naturally from the following design specifications:

Preserve polynomials. To preserve polynomial trends in the signal through scale, we should project onto real-valued scaling functions, which implies a standard real-valued lowpass filter $H$.

Hilbert transform. We wish to be able to distinguish high positive frequencies from high negative frequencies. Then, the real and imaginary parts of the resulting complex wavelets will be close to a Hilbert transform pair. This necessitates two separate complex high-pass filters.

Non-redundant. It is simple to design a complex filterbank that is non-redundant for a complex-valued input signal (taking an $N$-point complex input signal and producing $N$ complex wavelet coefficients). But, to also be non-redundant for a real-valued signal (taking an $N$-point real input signal and producing $N / 2$ complex wavelet coefficients) requires a special symmetry between the two complex high-pass filters such that one can be removed in the case of real signals (see Fig. 2). We will show that this implies a complex conjugate symmetry between the two complex highpass filters.

Orthogonal. We wish all three bands to be orthogonal.

FIR. To ensure good locality, we desire all filters to have a finite impulse response (FIR).

The primary result of this paper is to show that the filterbank satisfying the above complex highpass filter can always be decomposed 
itself as a two-stage filter bank, in which the first stage consists of a real-valued highpass filter and subsampler, and the second stage contains a complex projection filter (approximate Hilbert transform). The complex projection filter and its complex conjugate form a separate filter bank and can be designed in isolation. We will show that these need a very special symmetry.

\section{ORTHOGONAL COMPLEX CONJUGATE FILTER BANKS}

\subsection{Filterbank stucture}

The filterbank in Fig. 1 is the foundation of our complex orthonormal transform. The structure is basically a three-band filterbank with unbalanced down/upsampling. The lowpass branch consists of a conventional real-valued polynomial-preserving lowpass filter $H(z)$ whose output is downsampled by two. The two highpass branches consist of two complex highpass filters $G(z)$ and $G^{*}(z)$, whose outputs are downsampled by four.

As motivated above, by design we impose a strict symmetry on the highpass filters such that for real-valued input signals, one of the branches may be discarded. The wavelet transform is obtained by iterating on the real lowpass branch.

\subsection{Factorization of $\downarrow_{4} G(z)$}

In order to show that the transform we propose is the unique solution to the formulation in the introduction, we will prove that for any orthogonal structure with unbalanced sampling operators such as that given in Fig. 1, the four times subsampled filters in the highpass bands can be factored into two consecutive filtering and sampling operations (see Fig. 3). It turns out that both highpass branches share the same initial highpass filter, which is the complementary filter of the lowpass filter. Moreover, the second filtering operation is an approximate Hilbert transform projection. Since both highpass branches share the same initial complementary filter, the later projection can be isolated from the iteration of the transform and hence be designed separately.

Theorem 1 Given any orthogonal filter bank structure with unbalanced sampling operators such as that shown in Fig. 1, the four times subsampled filters can be factored into two consecutive filtering and sampling operations.

Proof: We start by rewriting the filterbank given in Fig. 1 into its most general form by substituting the orthogonal FIR fil-

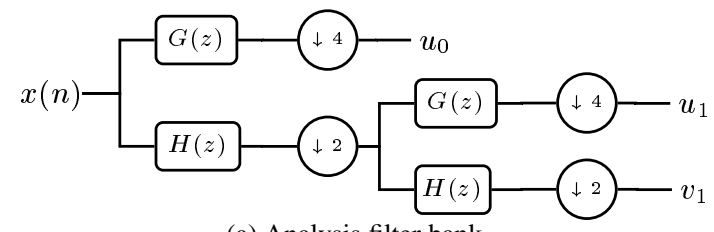

(a) Analysis filter bank

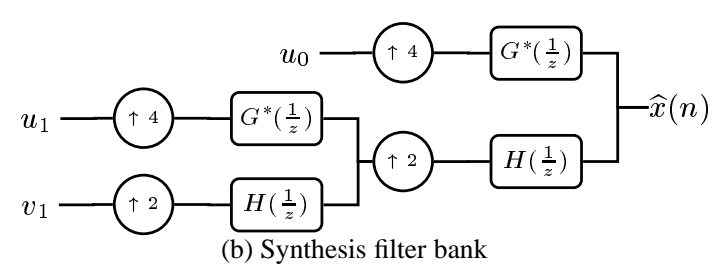

Fig. 2. Analysis and synthesis filter banks with real input signal $x(n)$. The band filtered by $G^{*}$ in Fig. 1 can be eliminated.

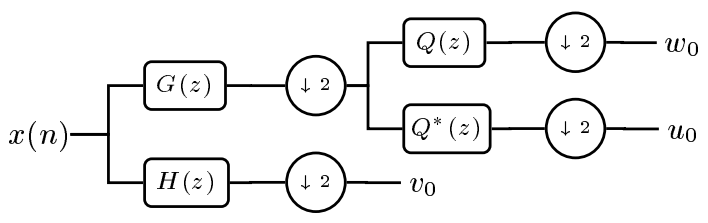

(a) Analysis filter bank

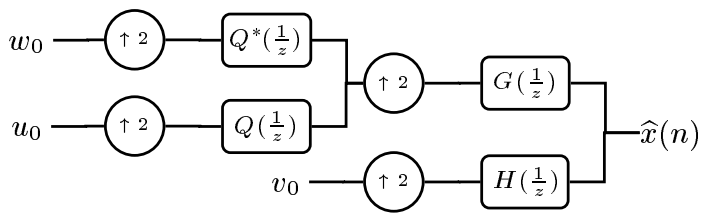

(b) Synthesis filter bank

Fig. 3. Analysis and synthesis filter banks after factoring each highpass branch. A real-valued input is assumed, so only one highpass channel is shown.

ters $F(z), G(z)$, and $H(z)$. After demultiplexing, we obtain the scheme given in Fig. 4.

Consider three inputs $u_{0}, u_{1}$, and $u_{2}$ and three outputs $\widehat{u}_{0}, \widehat{u}_{1}$, and $\widehat{u}_{2}$. For the filterbank to be a perfect reconstruction filterbank, the input has to equal the output

$$
\widehat{u}_{i}=u_{i}, \text { for } i=0,1,2
$$

for every triplet $\left(u_{0}, u_{1}, u_{2}\right)$. Choosing $u_{2}=u_{0}=0$ and $u_{1}=1$, we obtain three equations for the three different branches. Consider the equation that corresponds to the branch that links $u_{1}$ with $\widehat{u}_{0}$

$$
\widetilde{G}(z) H(z)+\widetilde{G}(-z) H(-z)=0 .
$$

We can rewrite (2) such that the first product equals an odd polynomial

$$
\widetilde{G}(z) H(z)=z Q_{1}\left(z^{2}\right)
$$

When we multiply both sides with $H(-z)$, (3) changes to

$$
\widetilde{G}(z) H(z) H(-z)=z H(-z) Q_{1}\left(z^{2}\right) .
$$

When we define $R\left(z^{2}\right)$ to be the polynomial $H(z) H(-z), \widetilde{G}(z)$ can be expressed as

$$
\widetilde{G}(z)=z H(-z) Q_{G}\left(z^{2}\right),
$$

with

$$
Q_{G}\left(z^{2}\right)=\frac{Q_{1}\left(z^{2}\right)}{R\left(z^{2}\right)}
$$

The same derivation follows when we choose $u_{1}=u_{0}=0$ and $u_{2}=1$, which yields an expression for the filter $\widetilde{F}(z)$

$$
\widetilde{F}(z)=z H(-z) Q_{F}\left(z^{2}\right),
$$

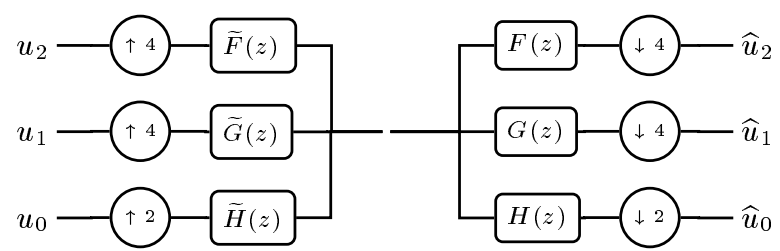

Fig. 4. Demultiplexed representation of generalized orthogonal structure shown in Fig. 1 after substituting the orthogonal filters $F(z), G(z)$, and $H(z)$. 


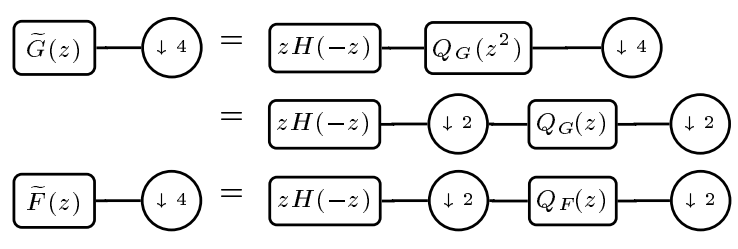

Fig. 5. Factoring the four times subsampled filters $\widetilde{G}(z)$ and $\widetilde{F}(z)$ into two concatenating filters using the Noble identities.

with

$$
Q_{F}\left(z^{2}\right)=\frac{Q_{2}\left(z^{2}\right)}{R\left(z^{2}\right)} .
$$

Both results (5) and (7) can be reinterpreted using the Noble identities as a concatenation of filter banks. This concept is shown in Fig. 5 for the filter (5). The lower branch in the same figure shows the result for (7). Both branches share the first filtering operation and hence can be factored into a common operation followed by a parallel filtering operation. The synthesis and analysis filters can be swapped as is obvious from Fig. 4. This shows that $G(z)$ and $F(z)$ can be factored in the same manner as $\widetilde{G}(z)$ and $\widetilde{F}(z)$.

Based on the proof we propose to use the filter bank in Fig. 3 for the iterative scheme to compute the complex wavelet transform. For real signals perfect reconstruction is then guaranteed because $w_{0}=u_{0}^{*}$ and hence the real part of the reconstructed signal $\widehat{x}(n)$ equals $1 / 2 x(n)$.

The design of the complex highpass filter $G(z)$ in Fig. 2 is now simplified to the design of a conventional real orthonormal filter bank structure and the orthogonal conjugate symmetric complex filter bank structure shown in Fig. 6 .

\subsection{Orthoconjugate constraints and implications}

In the previous section we showed that the decimate-by-four highpass branches in the complex filter bank of Fig. 1 can be factored into a real-valued filter followed by two projections. We now focus on the design of the projection filters $Q_{G}$ and $Q_{F}$.

Earlier we argued that conjugate symmetry is needed for perfect reconstruction of real-valued signals. The structure for the design of the orthoconjugate filter bank is shown in Fig. 6. We have substituted the conjugate filters $Q(z)$ and $Q^{*}(z)$ for $Q_{G}$ and $Q_{F}$. Using the general matrix formulation of [7] for the orthonormal filter bank of Fig. 6

$$
\left(\begin{array}{rr}
Q(z) & Q(-z) \\
Q^{*}(z) & Q^{*}(-z)
\end{array}\right)\left(\begin{array}{rr}
Q^{*}\left(\frac{1}{z}\right) & Q^{*}\left(-\frac{1}{z}\right) \\
Q\left(\frac{1}{z}\right) & Q\left(-\frac{1}{z}\right)
\end{array}\right)^{T}=2 I .
$$

We can derive the general representation for the class of filters with the correct symmetry. It turns out that any orthoconjugate FIR filter $Q(z)$ can be obtained from an orthonormal filter $U(z)$ with half sample symmetry

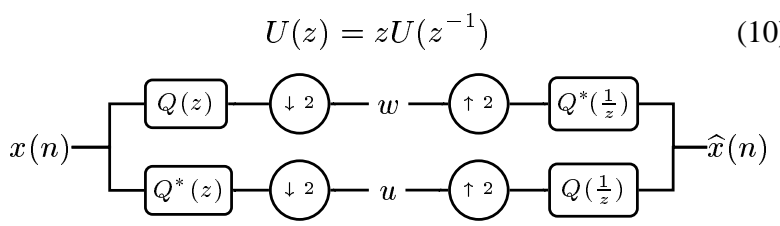

Fig. 6. The orthogonal complex conjugate filter bank for distinction of positive and negative frequencies can be designed separately.
Table 1. Filter coefficients $q(n)$ of the $\bar{D}_{2}, \bar{D}_{6}$, and $\bar{D}_{10}$ orthoconjugate complex Daubechies filters. $N$ indicates the filterlength.

\begin{tabular}{|r|r|rr|}
\hline$N$ & $n$ & \multicolumn{2}{|c|}{$q(n)$} \\
\hline \hline 2 & 0 & 0.70710678118655 & $0 . j$ \\
& 1 & 0. & $+0.70710678118655 j$ \\
\hline 6 & 0 & -0.06629126073624 & $-0.08558164961018 j$ \\
& 1 & 0.08558164961018 & $+0.11048543456040 j$ \\
& 2 & -0.66291260736239 & $-0.17116329922036 j$ \\
& 3 & 0.17116329922036 & $-0.66291260736239 j$ \\
& 4 & 0.11048543456040 & $-0.08558164961018 j$ \\
& 5 & 0.08558164961018 & $-0.06629126073624 j$ \\
\hline 10 & 0 & 0.01049245051230 & $+0.02059043708702 j$ \\
& 1 & -0.00872852869034 & $-0.01712890812780 j$ \\
& 2 & 0.08063970414533 & $+0.11794747353812 j$ \\
& 3 & -0.09422365674476 & $-0.15137970843150 j$ \\
& 4 & 0.64300323451588 & $+0.18285216450551 j$ \\
& 5 & -0.18285216450551 & $+0.64300323451588 j$ \\
& 6 & -0.15137970843150 & $+0.09422365674476 j$ \\
& 7 & -0.11794747353812 & $+0.08063970414533 j$ \\
& 8 & -0.01712890812780 & $+0.00872852869034 j$ \\
& 9 & -0.02059043708702 & $+0.01049245051230 j$ \\
\hline \hline
\end{tabular}

by a simple shift of $\pi / 2$ in frequency

$$
Q(z)=U(-j z)
$$

up to an even delay. Besides Haar's rectangular filters and the sinc, well-known filters that belong to the first class of filters are the complex Daubechies solutions [1],[2]. These are in a sense the shortest FIR solutions with the required half-sample symmetry. Table 1 gives the coefficients of the orthoconjugate complex Daubechies $\bar{D}_{2}, \bar{D}_{6}$, and $\bar{D}_{10}$ filters, where the overbar symbol reflects the orthoconjugate property of the filter. $\bar{D}_{2}$ can be interpreted as the "lazy" filter which merely splits even and odd samples. The $\bar{D}_{10}$ orthoconjugate complex Daubechies filter is illustrated in Fig. 7. Suppression of negative frequency with these filters is accomplished by vanishing moments at $\omega=-\pi / 2$.

\section{EXAMPLES}

While we have focussed on designing non-redundant orthoconjugate filter banks that can be used to compute the complex wavelet

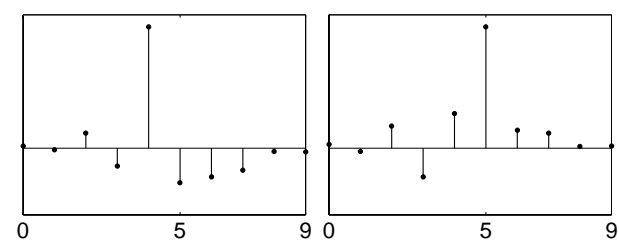

$\begin{array}{ll}\text { (a) } \mathcal{R} e\left\{\bar{D}_{10}\right\} & \text { (b) } \mathcal{I} m\left\{\bar{D}_{10}\right\}\end{array}$

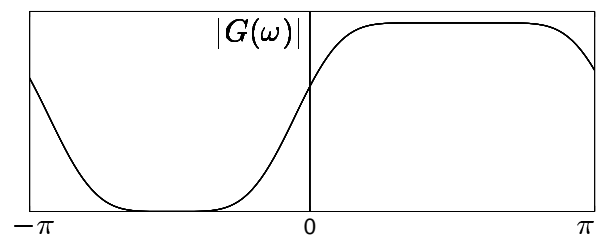

(c) $\mathcal{F}\left\{\bar{D}_{10}\right\}$

Fig. 7. The orthoconjugate complex Daubechies- $\bar{D}_{10}$ filter. (a) Real part, (b) imaginary part, and (c) magnitude of Fourier response. 


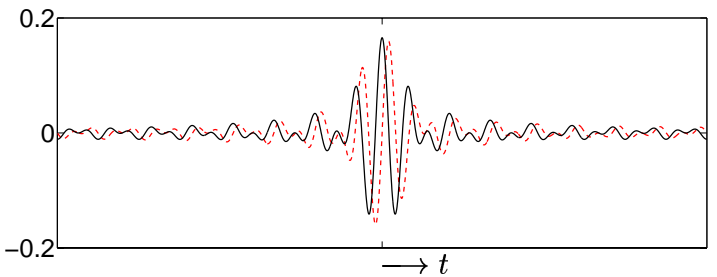

(a) Sinc-sinc

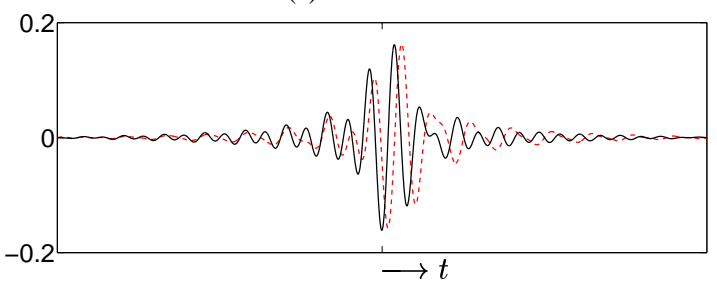

(b) Sinc- $\bar{D}_{10}$

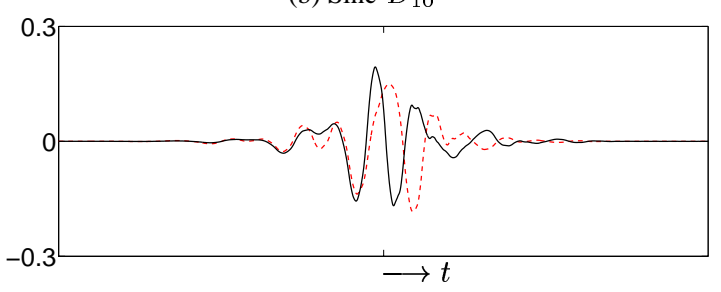

(c) $D_{10}-\bar{D}_{10}$

Fig. 8. Complex wavelets computed with (a) combination of sinc lowpass filter and sinc conjugate filter, (b) combination of sinc and $\bar{D}_{10}$ and (c) combination of Daubechies-10 and $\bar{D}_{10}$. Solid black line represents $\mathcal{R} e\{\psi(t)\}$, solid red line represents $\mathcal{I} m\{\psi(t)\}$

transform, we can also study the corresponding complex orthonormal basis functions. Using the cascade algorithm [7] we computed the wavelets in Fig. 8. These examples illustrate the effect of complex projection on the wavelets. Fig. 8 (a) shows the sinc lowpass-sinc Hilbert projection wavelet (sinc-sinc for short); the real wavelet is depicted in solid black and the imaginary wavelet in dashed red. In Fig. 8 (b) we show the sinc- $\bar{D}_{10}$ wavelet. In the iterative scheme we employed a sinc lowpass filter, for the Hilbert projection filter we used the $\bar{D}_{10}$-filter. In (c) we depicted the wavelet obtained from a $D_{10}-\bar{D}_{10}$ iterative scheme. On the right in Fig. 9 we show their respective Fourier magnitude responses. As expected, the sinc-sinc has perfectly suppressed negative frequencies, where energy remains present in the negative frequency range after imperfect suppression with $\bar{D}_{10}$.

\section{CONCLUSIONS}

In this paper we have proposed a new complex wavelet transform with orthonormal bases. The simple structure permits simple design criteria for two types of FIR filter banks; a general multiresolution filter bank and a conjugate symmetric filter bank with Hilbert transform properties.

Due to its non-redundancy for real-valued and complex signals the transform is promising for applications such as compression and related problems where this property is crucial and amplitude and phase play a pivotal role.

Although we impose a Hilbert transform relationship on the imaginary and real part of the conjugate filters, the complex wavelets show substantial aliasing energy in the negative fre-

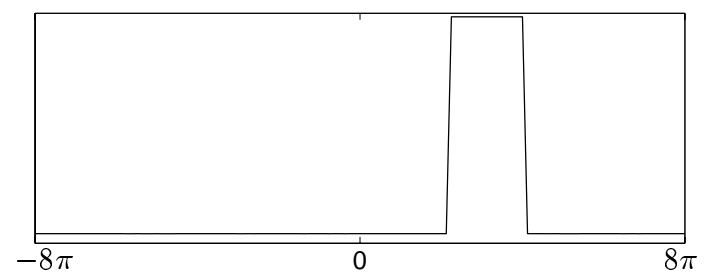

(a) Sinc-sinc

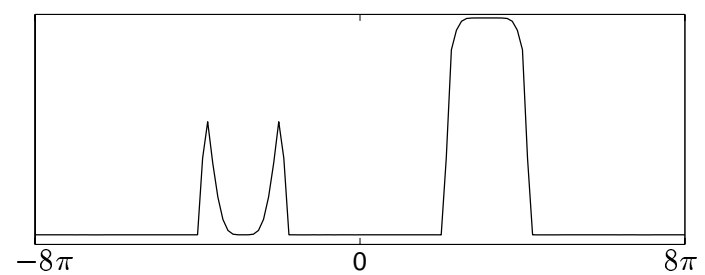

(b) Sinc- $\bar{D}_{10}$

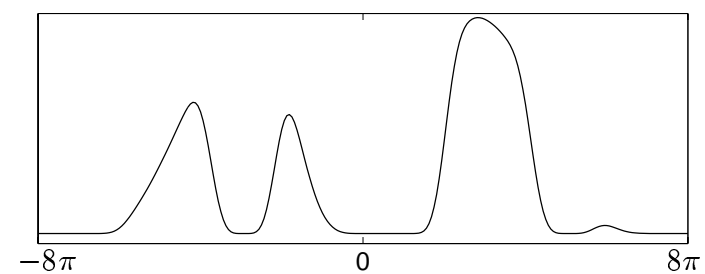

(c) Sinc- $\bar{D}_{10}$

Fig. 9. The Fourier responses of the complex wavelets in Fig. 8 computed with (a)combination of sinc lowpass filter and sinc conjugate filter, (b) combination of sinc and $\bar{D}_{10}$ and (c) combination of Daubechies-10 DWT and $\bar{D}_{10}$.

quency range which may effect the reliability of amplitude and phase information. Better suppression of the aliasing terms and improvement of the phase may require different filter design techniques such as biorthogonal filters which lead to symmetric linear phase wavelets.

\section{REFERENCES}

[1] W. Lawton, "Applications of complex valued wavelet transforms to subband decomposition," IEEE Trans. Sig. Proc., vol. 41, pp. 3566-3568, 1993.

[2] J. M. Lina and M. Mayrand, "Complex Daubechies wavelets," Appl. Comp. Harm. Anal., pp. 219-229, 1995.

[3] N. G. Kingsbury, "Complex wavelets for shift invariant analysis and filtering of signals," Appl. Comp. Harm. Anal., vol. 10, no. 3, pp. 234-253, 2001.

[4] I. W. Selesnick, "Hilbert transform pairs of wavelet bases," IEEE Signal Processing Letters, vol. 8, no. 6, 2001.

[5] R. L. C. van Spaendonck, F. C. A. Fernandes, M. Coates, and C. S. Burrus, "Non-redundant, directionally selective, complex wavelets," in IEEE Proc. Int. Conf. Image Proc., Extended Abstracts II. 2000, pp. 379-382, IEEE.

[6] F. C. A. Fernandes, I. W. Selesnick, R. L. C. van Spaendonck, and C. S. Burrus, "Complex wavelet transforms with allpass filters," EURASIP, 2002, submitted.

[7] M. Vetterli and J. Kovacevic, Wavelets and Subband Coding, Prentice Hall, Englewood Cliffs, NJ, 1995. 